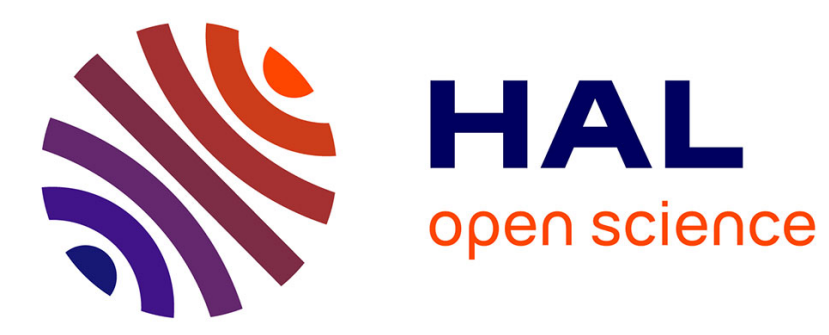

\title{
Development of the Corrected Force Analysis Technique for laminated composite panels
}

\author{
Fabien Marchetti, Kerem Ege, Quentin Leclere
}

\section{To cite this version:}

Fabien Marchetti, Kerem Ege, Quentin Leclere. Development of the Corrected Force Analysis Technique for laminated composite panels. Journal of Sound and Vibration, 2021, 490, pp.115692. 10.1016/j.jsv.2020.115692 . hal-02939456

\section{HAL Id: hal-02939456 \\ https://hal.science/hal-02939456}

Submitted on 25 Sep 2020

HAL is a multi-disciplinary open access archive for the deposit and dissemination of scientific research documents, whether they are published or not. The documents may come from teaching and research institutions in France or abroad, or from public or private research centers.
L'archive ouverte pluridisciplinaire HAL, est destinée au dépôt et à la diffusion de documents scientifiques de niveau recherche, publiés ou non, émanant des établissements d'enseignement et de recherche français ou étrangers, des laboratoires publics ou privés. 


\title{
Development of the Corrected Force Analysis Technique for laminated composite panels
}

\author{
Fabien Marchetti * \\ Matelys - Research Lab, F-69120 Vaulx-en-Velin, France \\ Kerem Ege \\ Univ Lyon, INSA-Lyon, LVA EA677, F-69621 Villeurbanne, France \\ Quentin Leclère \\ Univ Lyon, INSA-Lyon, LVA EA677, F-69621 Villeurbanne, France
}

\begin{abstract}
The aim of this paper is to propose a methodology for identifying the pressure field applied on a laminated composite plate from its measured vibration response. The general framework of this study is the Corrected Force Analysis Technique (CFAT), which was previously used on isotropic plates. This method estimates the spatial derivatives of the equation of motion with a finite difference scheme and corrects the bias error generated by this approximation. The paper presents an extension of this technique to deal with laminated panels. In a first instance, the finite difference scheme has been expanded to allow the identification of the 5 spatial derivatives that compose the equation of motion of such structures. The correction, considered in the wavenumber domain, has also been adapted and is now calculated numerically. In a second instance, the effect of measurement noise has been treated with the implementation of a regularisation technique based on the adaptation of the size of the scheme as function of the frequency. Numerical simulations and contact-less measurements have been conducted on different carbon fibers composite plates. Results are presented in terms of identified pressure fields and input force magnitude.
\end{abstract}

Key words: Inverse method; source identification; pressure field; laminated composite plate; CFAT method; FAT method;

\footnotetext{
* corresponding author

Email addresses: fabien.marchetti@matelys.com (Fabien Marchetti), kerem.egedinsa-lyon.fr(Kerem Ege), quentin. lecleredinsa-lyon.fr
} (Quentin Leclère). 


\section{Introduction}

The identification of vibration sources is a large topic widely addressed in the scientific and industrial community. Several identification techniques have been published in the literature and the development of new complex materials brings us to adapt them.

One concept of force identification is the process of determining applied loadings from the system response, it belongs to the class of inverse problems [1|2]. Contrarily to the direct measurement by means of force transducers, the structure is used as its own force sensor. The inverse problem requires a fine knowledge of the dynamic characteristics of the system and accurate measured data. This approach was firstly introduced by pioneer works about transfer path analysis [3/415]. Some limitations arised from these techniques: the ill-conditioning of the transfer matrix as well as the insufficient accuracy of the measured mobility often gave poor results in terms of force quantification. Different regularization techniques have been developed to reduce these errors: weighted least square procedures [6/718], generalized inverse [9], Tikhonov regularization [10]11].

Localization of sources has been introduced with the structural intensity which details the power flowing in the structure [12]13]. This technique has been combined with a high wavenumber filtering to reduce the effect of measurement noise [14]. More recently, an alternative method called VFM (Virtual Field Method) uses the principle of virtual works to identify mechanical parameters of the system [15]. Various applications of this method have been published in the domain of source identification such as mechanical point loads on bending plates [16], spatially correlated excitations [17] or a time-resolved identification [18].

The Force Analysis Technique (FAT) is an experimental method of pressure field reconstruction, developed for beams [19], plates [20] and shells [21]. The principal strength of FAT is its capacity to described the structure's behaviour with a local analytical model. The local aspect does not require any knowledge outside the studied area (boundary conditions, sources) and the analytical model provides the possibility to develop the method with different theories. The equation of motion is approximated by a finite different scheme applied on the measured displacement field. In practice, this approximation implies two main sources of error in the reconstruction process. The first one is a singularity of the finite differences scheme response in the wavenumber domain. Another approach, CFAT (Corrected FAT) [22] attenuates this singularity by adding correction factors in the derivative assessments. The second source of errors is the sensitivity to measurement uncertainties. Two regularisation techniques have been adopted to avoid this instability: a low-pass wavenumber filtering by FAT [19|20] and an adapted spacing of the scheme by CFAT [23]. FAT and CFAT have also been extended for material characterisation issues, for the cases of thin isotropic beams and plates (CFAT) [24], thin 
orthotropic structures (FAT) [25] and thick beams (FAT) [26].

Considering the advent of new complex materials such as composites [27], this paper proposes an extension of the CFAT method for the identification of sources on laminated plates. This type of structures is constituted from several orthotropic layers with different orientation angles. This paper aims at adapting the CFAT regularization technique to laminated plates using a numerical approach for the determination of the correction factors.

The first section of this paper describes the methodology. The performance of the CFAT correction is illustrated on a numerical application using the finite element software Nastran (MSC software). In a second part, the effect and tackling of measurement noise is addressed. In the last section, Laser Doppler Vibrometer (LDV) measurements on two composite plates with different layups are realized. The results are presented in terms of reconstructed pressure fields and force quantification.

\section{Methodology}

\subsection{Equation of motion of a laminated composite plate}

The methodology has been developed assuming that the material characteristics of the laminated plate are known (orthotropic rigidities and orientation angles of each layer). An equivalent approach is used to model the response of the multilayer plate [28]. This model calculates analytically at a given frequency the flexural rigidities of an equivalent single layer anisotropic plate. Once these equivalent parameters have been obtained, the Love-Kirchhoff equation of motion is used to write the harmonic local equilibrium of the plate:

$D_{11} \frac{\partial^{4} w}{\partial x^{4}}+D_{22} \frac{\partial^{4} w}{\partial y^{4}}+D_{12} \frac{\partial^{4} w}{\partial x^{2} \partial y^{2}}+D_{16} \frac{\partial^{4} w}{\partial x^{3} \partial y}+D_{26} \frac{\partial^{4} w}{\partial x \partial y^{3}}-\rho h \omega^{2} w(x, y)=p(x, y)$,

where the coefficients $D_{i j}, w, p$ and $\omega$ represent the flexural rigidities of the equivalent anisotropic plate, the displacement, the pressure fields and the angular frequency, respectively. $\rho h$ is the mass per unit area of the equivalent plate. Contrarily to orthotropic structures for which only 3 flexural rigidities are needed $\left(D_{11}, D_{22}, D_{12}\right)$, the rigidities $D_{16}, D_{26}$ are not null for the general case of anisotropic plates, because of the possible flexural-torsion interaction.

In the wavenumber domain, Eq. (1) writes

$$
\left(D_{11} k_{x}^{4}+D_{22} k_{y}^{4}+D_{12} k_{x}^{2} k_{y}^{2}+D_{16} k_{x}^{3} k_{y}+D_{26} k_{x} k_{y}^{3}-\rho h \omega^{2}\right) \widehat{w}\left(k_{x}, k_{y}\right)=\widehat{p}\left(k_{x}, k_{y}\right),
$$


with $\widehat{w}\left(k_{x}, k_{y}\right), \widehat{p}\left(k_{x}, k_{y}\right)$ the 2D Fourier transforms of $w(x, y)$ and $p(x, y)$. Considering a polar reference system $(k, \theta)$ of the wavenumber plane, with $k_{x}=k \cos (\theta)$ and $k_{y}=k \sin (\theta)$, one obtains

$$
\left(k^{4}\left(D_{11} c^{4}+D_{22} s^{4}+D_{12} c^{2} s^{2}+D_{16} c^{s}+D_{26} c s^{3}\right)-\rho h \omega^{2}\right) \widehat{w}(k, \theta)=\widehat{p}(k, \theta),
$$

with $c=\cos (\theta)$ and $s=\sin (\theta)$. A natural wavenumber is easily expressed for each angle $\theta$ and frequency $\omega$ :

$$
k_{N}^{4}(\omega, \theta)=\frac{\rho h \omega^{2}}{D_{11} c^{4}+D_{22} s^{4}+D_{12} c^{2} s^{2}+D_{16} c^{s}+D_{26} c s^{3}} .
$$

\subsection{Classic FAT theory for anisotropic plates}

The FAT method estimates the pressure field by approximating the partial derivatives of the equation of motion (1) with a finite difference scheme applied to the displacement field [25]:

$$
\begin{gathered}
\frac{\partial^{4} w}{\partial x^{4}} \approx \delta^{4 x}=\frac{1}{\Delta_{x}^{4}}\left(w_{+4,0}-4 w_{+2,0}+6 w_{0,0}-4 w_{-2,0}+w_{-4,0}\right), \\
\frac{\partial^{4} w}{\partial y^{4}} \approx \delta^{4 y}=\frac{1}{\Delta_{y}^{4}}\left(w_{0,+4}-4 w_{0,+2}+6 w_{0,0}-4 w_{0,-2}+w_{0,-4}\right), \\
\frac{\partial^{4} w}{\partial x^{2} \partial y^{2}} \approx \delta^{2 x 2 y}=\frac{1}{\Delta_{x}^{2} \Delta_{y}^{2}}\left(w_{+2,+2}+w_{+2,-2}+w_{-2,+2}+w_{-2,-2}\right. \\
\left.-2\left(w_{+2,0}+w_{-2,0}+w_{0,+2}+w_{0,-2}\right)+4 w_{0,0}\right), \\
\frac{\partial^{4} w}{\partial x^{3} \partial y} \approx \delta^{3 x y}=\frac{1}{\Delta_{x}^{3} \Delta_{y}}\left(w_{+3,+1}-w_{+3,-1}-w_{-3,+1}+w_{-3,-1}\right. \\
\left.-3\left(w_{+1,+1}-w_{+1,-1}-w_{-1,+1}+w_{-1,-1}\right)\right), \\
\frac{\partial^{4} w}{\partial x \partial y^{3}} \approx \delta^{x 3 y}=\frac{1}{\Delta_{x} \Delta_{y}^{3}}\left(w_{+1,+3}-w_{-1,+3}-w_{+1,-3}+w_{-1,-3}\right. \\
\left.-3\left(w_{+1,+1}-w_{+1,-1}-w_{-1,+1}+w_{-1,-1}\right)\right),
\end{gathered}
$$

where

$$
w_{\alpha, \beta}=w\left(x+\frac{\alpha \Delta_{x}}{2}, y+\frac{\beta \Delta_{y}}{2}\right) .
$$

The finite difference scheme is finally composed of 25 points (see Fig. 11). $\Delta_{x}$ and $\Delta_{y}$ represent the spacing between two consecutive points of the scheme in the $x$ and 
$y$ axes respectively. A regular cartesian measurement grid, defined by the spatial steps $d_{x}$ and $d_{y}$, is considered. The pressure field is obtained by scanning the grid with the finite difference scheme. In this configuration of the scheme, $\Delta_{x}=2 d_{x}$ and $\Delta_{y}=2 d_{y}$.

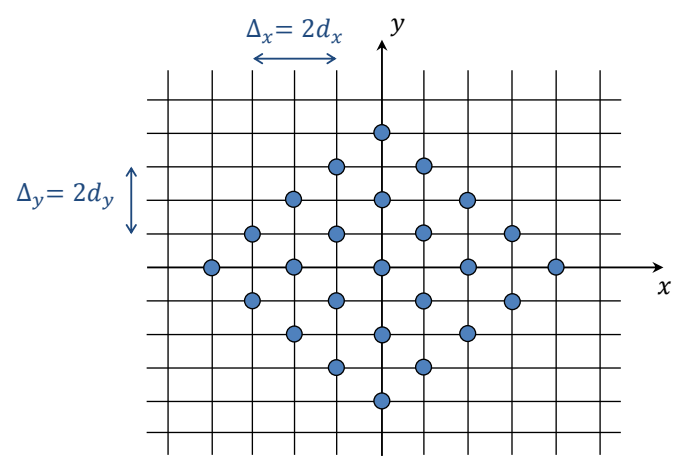

Fig. 1. Finite difference scheme of FAT and CFAT for the general anisotropic case.

Finally, the pressure field estimated by FAT is:

$$
p^{\mathrm{FAT}}(x, y)=D_{11} \delta^{4 x}+D_{22} \delta^{4 y}+D_{12} \delta^{2 x 2 y}+D_{16} \delta^{3 x y}+D_{26} \delta^{x 3 y}-\rho h \omega^{2} w(x, y)
$$

Note that for the particular case $d_{y}=d_{x}$, the finite difference scheme can be rotated by $\pi / 4$, and scaled by a factor $1 / \sqrt{2}$, with a resulting spatial step of the scheme equal to $\Delta_{x}=\Delta_{y}=\sqrt{2} d_{x}$ (the reader can refer to [25] for further details about this particular case). In the present work, the general case $d_{y} \neq d_{x}$ is considered because it can be well adapted when dealing with anisotropic structures, that can exhibit strongly different natural wavenumbers on $x$ and $y$ axes (and more generally in any direction $\theta$ ).

The performance of the method is classically studied in the literature as a function of $n$ the number of points of the finite difference scheme by structural wavelength, ratio between $\lambda_{N}$ and $\Delta$ in the case of isotropic plates. For the general anisotropic case, the definition of this parameter is more complex, because the step of the scheme is defined in two directions $\left(\Delta_{x}\right.$ and $\left.\Delta_{y}\right)$ while the structural wavelength depends on the wave direction $\theta$. In this work, the following conventions are used:

$n_{x}(\omega)=\frac{2 \pi}{\underset{\theta}{\operatorname{argmax}}\left(k_{\mathrm{N}}(\omega, \theta) \cos (\theta)\right) \Delta_{x}} \quad ; \quad n_{y}(\omega)=\frac{2 \pi}{\underset{\theta}{\operatorname{argmax}}\left(k_{\mathrm{N}}(\omega, \theta) \sin (\theta)\right) \Delta_{y}}$,

that corresponds to the half width and heigth of the rectangle in which the $k_{N}(\theta)$ parametric curve is inscribed. 


\subsection{Wavenumber response of FAT for laminated plates}

The FAT estimation of the pressure field in the wavenumber domain is written as follows:

$\widehat{p}^{\mathrm{FAT}}\left(k_{x}, k_{y}\right)=\left(D_{11} \widehat{\delta}^{4 x}+D_{22} \widehat{\delta}^{4 y}+D_{12} \widehat{\delta}^{2 x 2 y}+D_{16} \widehat{\delta}^{3 x y}+D_{26} \widehat{\delta}^{x 3 y}-\rho h \omega^{2}\right) \widehat{w}\left(k_{x}, k_{y}\right)$,

with $\widehat{p}^{\mathrm{FAT}}$ the 2D Fourier transform of $p^{\mathrm{FAT}}(x, y)$, and where the coefficients $\widehat{\delta}^{i j}$ correspond to the 2D Fourier transforms of the finite difference schemes and are expressed as follows:

$$
\begin{aligned}
\widehat{\delta}^{4 x} & =\frac{16 \sin ^{4}\left(\frac{k_{x} \Delta_{x}}{2}\right)}{\Delta_{x}^{4}}, \\
\widehat{\delta}^{4 y} & =\frac{16 \sin ^{4}\left(\frac{k_{y} \Delta_{y}}{2}\right)}{\Delta_{y}^{4}}, \\
\widehat{\delta}^{2 x 2 y} & =\frac{16 \sin ^{2}\left(\frac{k_{x} \Delta_{x}}{2}\right) \sin ^{2}\left(\frac{k_{y} \Delta_{y}}{2}\right)}{\Delta_{x}^{2} \Delta_{y}^{2}}, \\
\widehat{\delta}^{3 x y} & =\frac{16 \sin ^{3}\left(\frac{k_{x} \Delta_{x}}{2}\right) \sin \left(\frac{k_{y} \Delta_{y}}{2}\right)}{\Delta_{x}^{3} \Delta_{y}}, \\
\widehat{\delta}^{x 3 y} & =\frac{16 \sin \left(\frac{k_{x} \Delta_{x}}{2}\right) \sin ^{3}\left(\frac{k_{y} \Delta_{y}}{2}\right)}{\Delta_{x} \Delta_{y}^{3}} .
\end{aligned}
$$

The ratio between identified and real load distributions in the wavenumber domain, representing the estimation error of the FAT method, can now be expressed as:

$$
E_{\mathrm{FAT}}\left(\omega, \Delta_{x}, \Delta_{y}, k, \theta\right)=\frac{\hat{p}^{\mathrm{FAT}}(k, \theta)}{\hat{p}(k, \theta)}=\frac{\mathcal{K}_{\mathrm{FAT}}^{4}-k_{\mathrm{N}}^{4}}{k^{4}-k_{\mathrm{N}}^{4}},
$$

where:

$$
\mathcal{K}_{\mathrm{FAT}}^{4}=\frac{D_{11} \widehat{\delta}^{4 x}+D_{22} \widehat{\delta}^{4 y}+D_{12} \widehat{\delta}^{2 x 2 y}+D_{16} \widehat{\delta}^{3 x y}+D_{26} \widehat{\delta}^{x 3 y}}{D_{11} c^{4}+D_{22} s^{4}+D_{12} c^{2} s^{2}+D_{16} c^{3} s+D_{26} c s^{3}} .
$$

As it has already been observed in the literature for isotropic plates [22], the FAT response $E_{\mathrm{FAT}}$ has a general low pass filtering effect in the wavenumber domain, with a strong singularity around $k=k_{N}$, due to the root of the denominator term. This observation is extended here to the general anisotropic case, for which the natural wavenumber depends on the angle $\theta$. Some illustrations are provided in Section 3 , 


\subsection{Corrected FAT for anisotropic plates}

As proposed in [22], the CFAT theory considers correction factors applied to the finite difference scheme of FAT in order to attenuate the bias error around the natural wavenumber of the plate. Expressions of correction factors are given in [22] for isotropic plates; a similar analysis is conducted in the present work to propose correction factors in the general anisotropic case.

Each coefficient of the scheme $\delta^{i j}$ is multiplied by a correction factor $\mu_{i j}(\omega)$ to be determined :

$$
\begin{aligned}
p^{\mathrm{CFAT}}(x, y)=D_{11} \mu_{11} \delta^{4 x}+D_{22} \mu_{22} \delta^{4 y}+D_{12} \mu_{12} \delta^{2 x 2 y}+D_{16} \mu_{16} \delta^{3 x y} \\
+D_{26} \mu_{26} \delta^{x 3 y}-\rho h \omega^{2} w(x, y)
\end{aligned}
$$

In a similar way to FAT, the wavenumber response of CFAT can also be defined as:

$$
E_{\mathrm{CFAT}}\left(\omega, \Delta_{x}, \Delta_{y}, k, \theta\right)=\frac{\widehat{p}^{\mathrm{CFAT}}(k, \theta)}{\widehat{p}(k, \theta)}=\frac{\mathcal{K}_{\mathrm{CFAT}}^{4}-k_{\mathrm{N}}^{4}}{k^{4}-k_{\mathrm{N}}^{4}}
$$

with:

$\mathcal{K}_{\mathrm{CFAT}}^{4}(k, \theta)=\frac{D_{11} \mu_{11} \widehat{\delta}^{4 x}+D_{22} \mu_{22} \widehat{\delta}^{4 y}+D_{12} \mu_{12} \widehat{\delta}^{2 x 2 y}+D_{16} \mu_{16} \widehat{\delta}^{3 x y}+D_{26} \mu_{26} \widehat{\delta}^{x 3 y}}{D_{11} c^{4}+D_{22} s^{4}+D_{12} c^{2} s^{2}+D_{16} c^{3} s+D_{26} c s^{3}}$.

The values of the correction factors are chosen to equalize as much as possible the root of the numerator with the one of the denominator of $E_{\text {CFAT }}$ in Eq. (19). For a given direction $\theta$, it brings:

$$
\mathcal{K}_{\mathrm{CFAT}}^{4}\left(k_{N}(\theta), \theta\right)=k_{N}^{4}
$$

The correction factors can be analytically identified by considering Eq. (20) for arbitrary values of $\theta$, as many values as the number of correction factors to be determined. In the isotropic case treated in [22], the values $\theta=0$ and $\theta=\pi / 4$ are arbitrarily chosen. For the anisotropic case, 5 different values of $\theta$ have to be considered to determine analytic expressions of the 5 correction factors (see [29]). Another possibility is to find numerically a least squares solution satisfying Eq. (20) for a number of directions $\theta$ much larger than the number of correction factors to be determined. This numerical approach relies on a linear system resulting from Eq. (20) written for $n$ values of $\theta$ (at least 5, but many more in practice so as to ensure the convergence of the results) from 0 to $\pi\left(k_{\mathrm{N}}(\theta)\right.$ being $\pi$ periodic for composite laminated structures):

$$
[\mathcal{B}]\left\{\mu_{i j}\right\}=\{\mathcal{C}\},
$$

where $\mu_{i j}$ are the unknown correction factors, and where: 


$$
[\mathcal{B}]=\left[\begin{array}{ccccc}
D_{11} X_{1}^{4} & D_{22} Y_{1}^{4} & D_{12} X_{1}^{2} Y_{1}^{2} & D_{16} X_{1}^{3} Y_{1} & D_{26} X_{1} Y_{1}^{3} \\
\vdots & \vdots & \vdots & \vdots & \vdots \\
D_{11} X_{n}^{4} & D_{22} Y_{n}^{4} & D_{12} X_{n}^{2} Y_{n}^{2} & D_{16} X_{n}^{3} Y_{n} & D_{26} X_{n} Y_{n}^{3}
\end{array}\right],\{\mathcal{C}\}=\rho h \omega^{2}\left(\begin{array}{c}
1 \\
\vdots \\
1
\end{array}\right)
$$

with

$$
X_{i}=\frac{2 \sin \left(k_{\mathrm{N}}\left(\theta_{i}\right) \cos \left(\theta_{i}\right) \Delta_{x} / 2\right)}{\Delta_{x}} \quad ; \quad Y_{i}=\frac{2 \sin \left(k_{\mathrm{N}}\left(\theta_{i}\right) \sin \left(\theta_{i}\right) \Delta_{y} / 2\right)}{\Delta_{y}} .
$$

This system is solved using standard least squares:

$$
\left\{\mu_{i j}\right\}=\left([\mathcal{B}]^{\mathrm{H}}[\mathcal{B}]\right)^{-1}[\mathcal{B}]^{\mathrm{H}}\{\mathcal{C}\}
$$

This numerical solution depends on the set of $n$ values of $\theta$ chosen to create the matrix $[\mathcal{B}]$, for instance by regularly sampling the interval $[0, \pi]$. However, one observes a convergence when $n$ tends to infinity. The effect of $n$ on the correction parameter determination and wavenumber response of CFAT is discussed and illustrated in Section 3.1 .

\section{Numerical validation}

In this section, FAT and CFAT are applied on simulated vibration displacement fields of laminated composite plates, generated using the frequency response analysis (Sol 111) of Nastran. These plates are composed of four carbon fibers layers mixed with resin and have been experimentally studied in a recent paper submitted to Journal of Sound and Vibration concerning the modelling of laminated composite plate and sandwich structure [28]. The first plate is orthotropic with fiber orientations of $0^{\circ}$ for each layer and the second one is anisotropic with fiber orientations of $60^{\circ}$ for the external layers and $-60^{\circ}$ for the internal layers. The characteristics of one layer are presented in Table 1 .

Table 1

\begin{tabular}{c|c|c|c|c|c}
$h(\mathrm{~m})$ & $\rho\left(\mathrm{kg} \cdot \mathrm{m}^{-3}\right)$ & $E_{x}(\mathrm{GPa})$ & $E_{y}(\mathrm{GPa})$ & $G_{x y}(\mathrm{GPa})$ & $\nu_{x y}(-)$ \\
\hline 0.187 & 1540 & 138 & 9.5 & 3.4 & 0.31
\end{tabular}

Characteristics of one layer of the simulated multilayer laminated plates.

A harmonic force, located at the center of the plates, is applied on the $0.5 \times 0.5 \mathrm{~m}^{2}$ plates with an amplitude of $1 \mathrm{~N}$. Simply supported boundary conditions are considered on the edges. The frequency range of the simulation is 1 to $10 \mathrm{kHz}$. The plates have been modeled with a $2 \mathrm{D}$ mesh of $250 \times 250$ nodes, with NASTRAN PCOMP laminated composite elements property. The equivalent flexural rigidities 
of both plates have been calculated with the analytical model described in [28] as function of frequency. This model considers, at one given frequency, a single layer anisotropic plate equivalent to the multilayer. The convergence of the results of Nastran has been numerically verified with a modal study from 9.9 to $10 \mathrm{kHz}$. The vibration field has been extracted on a regular mesh $d_{x}=d_{y}=4 \mathrm{~mm}$ to simulate a measurement mesh respecting Shannon's criterion up to $10 \mathrm{kHz}$.

\subsection{Wavenumber responses of FAT and CFAT, calculation of correction factors}

The wavenumber responses of FAT (Eq. (17)) and CFAT (Eq. (19) have been calculated and compared at $10 \mathrm{kHz}$. The equivalent flexural rigidities of both plates at this frequency are presented in Tables 2 and 3 .

Table 2

\begin{tabular}{c|c|c|c|c}
$D_{11}$ (N.m) & $D_{22}$ (N.m) & $D_{12}$ (N.m) & $D_{16}$ (N.m) & $D_{26}$ (N.m) \\
\hline 4.57 & 0.33 & 0.66 & 0 & 0
\end{tabular}

Equivalent flexural rigidities of the $0^{\circ} / 0^{\circ} / 0^{\circ} / 0^{\circ}$ composite plate at $10 \mathrm{kHz}$, according to [28].

Table 3

\begin{tabular}{c|c|c|c|c}
$D_{11}$ (N.m) & $D_{22}$ (N.m) & $D_{12}$ (N.m) & $D_{16}$ (N.m) & $D_{26}$ (N.m) \\
\hline 0.55 & 2.68 & 5.54 & 1.30 & 4.19
\end{tabular}

Equivalent flexural rigidities of the $60^{\circ} /-60^{\circ} /-60^{\circ} / 60^{\circ}$ composite plate at $10 \mathrm{kHz}$, according to [28].

The correction factors have been calculated using the least squares method presented in section 2.4 with 100 equations, for 100 values of $\theta$, regular sampling between 0 and $\pi$. The wavenumber responses of the two methods, as given by Eq. (17) and (19) are drawn in Figures 2 and 3 as function of $k_{x}$ and $k_{y}$. The singularity is clearly observed on FAT responses around $k=k_{\mathrm{N}}$. The CFAT correction efficiently attenuates it but can not completely remove it totally since the correction factors do not depend on the direction.

The convergence of the least squares method used to calculate correction factors has been studied. Figure 4 shows the calculated values of the correction factors as function of the number of equations in Eq. (23), divided by the values found with 1000 equations. The estimations given by the least square method are erratic up to 20 equations. Above, the relative error decreases gradually and becomes very low: less than $1 \%$ between 100 and 1000 equations. Note that the same trend is observed at other frequencies; a consequence of it is the continuity observed on the frequency dependence of the correcting factors observed in Figure 5. From this observation, we can assume that a number of 100 equations seems to be a good compromise between convergence and time consumption of the method. 
(a) FAT

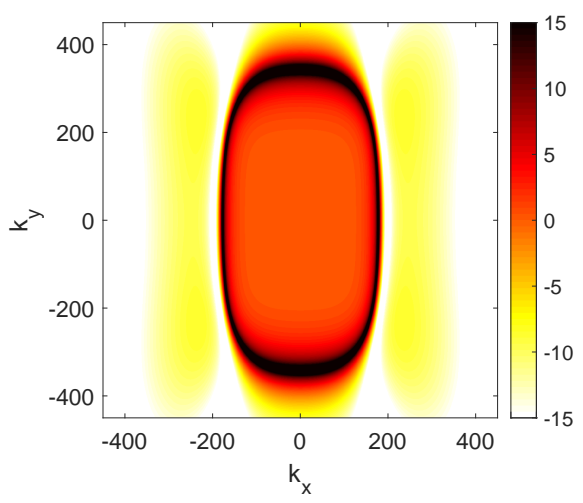

(b) CFAT

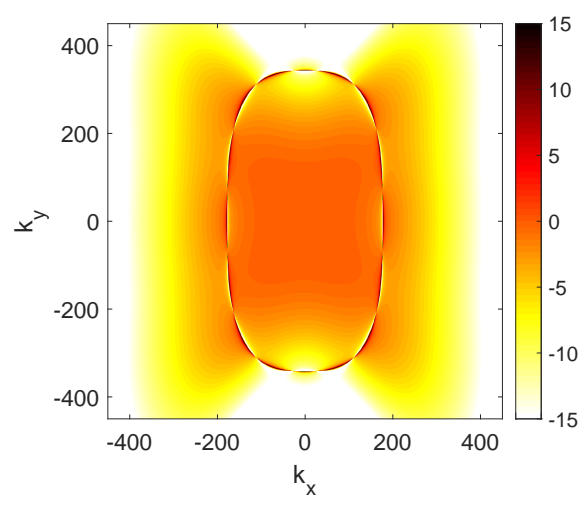

Fig. 2. Wavenumber responses (dB) of FAT (a) and CFAT (b) with numerical correction factors (100 equations) for orthotropic flexural rigidities with $n_{x}=4.42$ and $n_{y}=2.29$.

(a) FAT

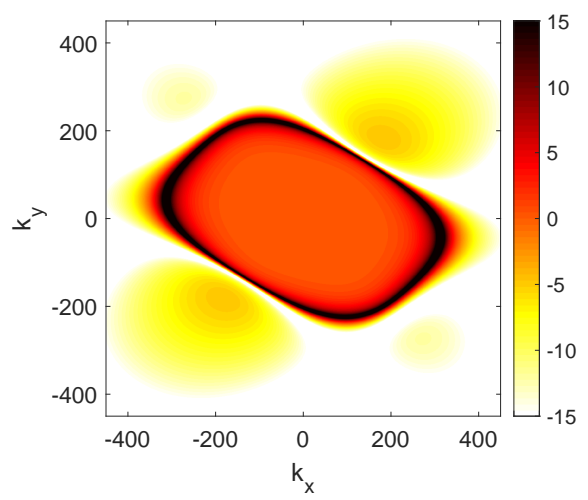

(b) CFAT

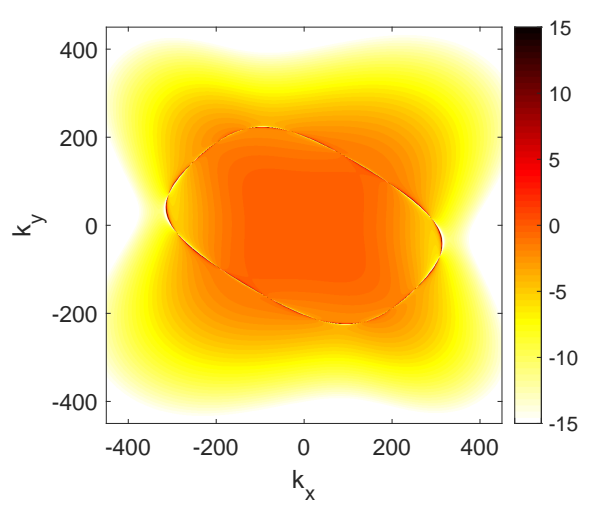

Fig. 3. Wavenumber responses (dB) of FAT (a) and CFAT (b) with numerical correction factors (100 equations) for laminated flexural rigidities with $n_{x}=2.61$ and $n_{y}=3.87$.

The number of points per wavelength is an essential criterion of the method. Indeed, at low frequencies (high values of $n_{x}$ and $n_{y}$ ), the estimation error of FAT is negligible and the correction factors tend to 1 (see Figure 5). When the frequency increases, the bias error becomes significant and the CFAT correction factors strongly deviate from 1 . As a rule of thumb, verified in previous work for the case of isotropic plates [23], the correction becomes necessary when the number of points per wavelength falls below 4 .

\subsection{Identified pressure fields}

In a second step, the load distributions estimated by FAT and CFAT have been compared for both plates. Figures 6 and 7 present the pressure fields integrated in 
(a) Orthotropic plate

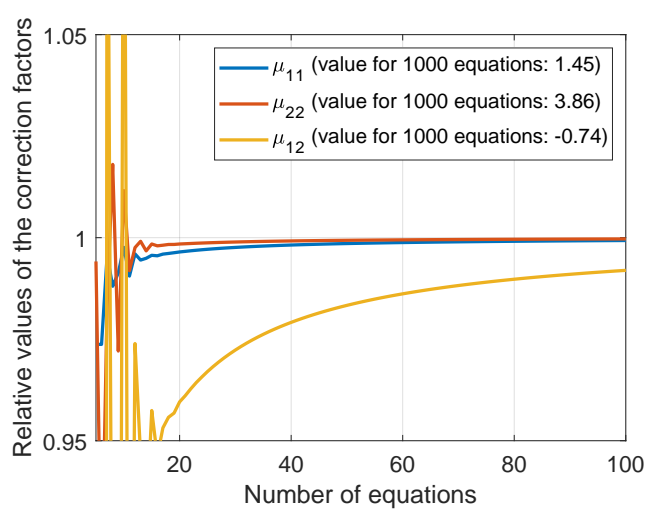

(b) Laminated plate

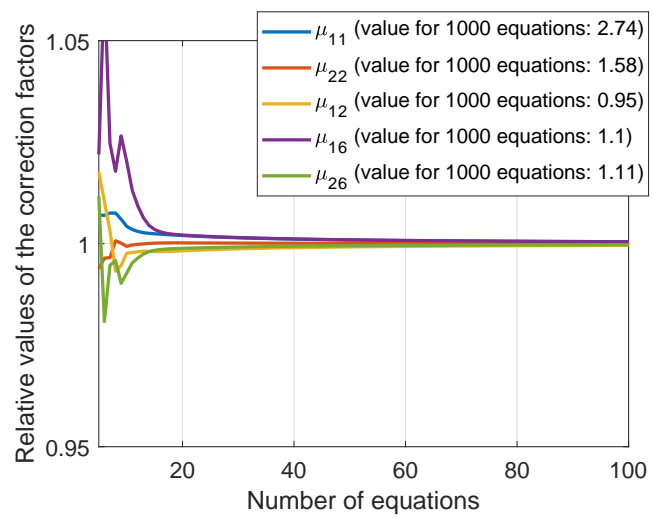

Fig. 4. Relative values of the correction factor at $10 \mathrm{kHz}$ as function of the number of equations. (a): orthotropic plate, (b): laminated plate.

(a)

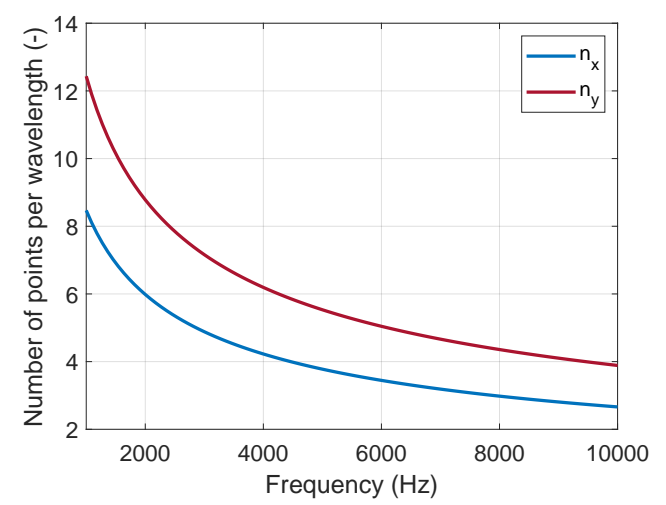

(b)

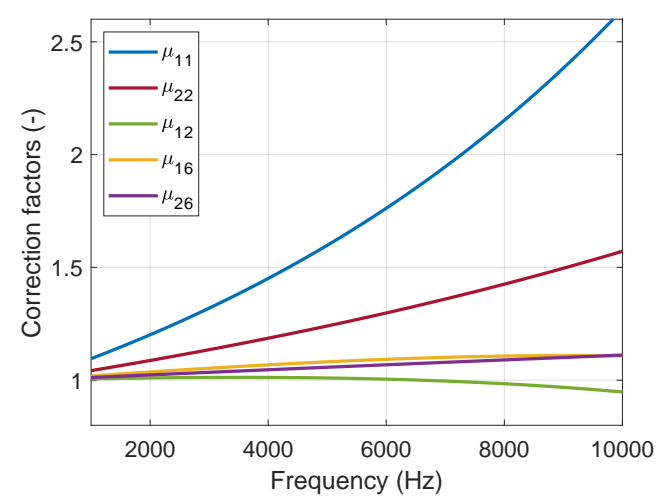

Fig. 5. Number of points per wavelength (a) and correction factors (b) calculated for the laminated plate with $\Delta_{x}=\Delta_{y}=4 \mathrm{~mm}$.

the whole frequency band.

FAT and CFAT give the exact location of the source. However, differences occurs between both methods in the non-loaded area: the pressure level estimated by FAT is relatively high. In this area, the energy is essentially governed by the resonances of the plate, when the wavenumber of the propagating wave is equal to the natural wavenumber of the plate $\left(k=k_{\mathrm{N}}\right)$. The singularity of FAT around this wavenumber amplifies the energy and the estimation error. The correction brought by CFAT strongly attenuates these residual forces. Futhermore, the load distribution around the excitation point extends to large area of approximately $7.6 \times 3.6 \mathrm{~cm}^{2}$ for both plates. This is due to the wavenumuber lowpass filtering effect of FAT and CFAT methods, whose response depends on $\theta$ and $\omega$. 
(a) FAT

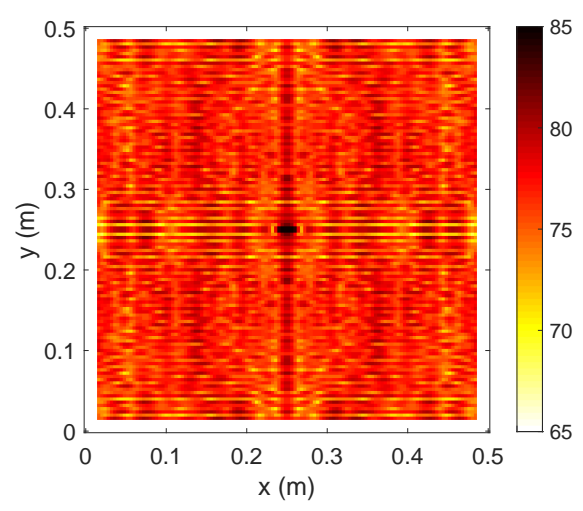

(b) CFAT

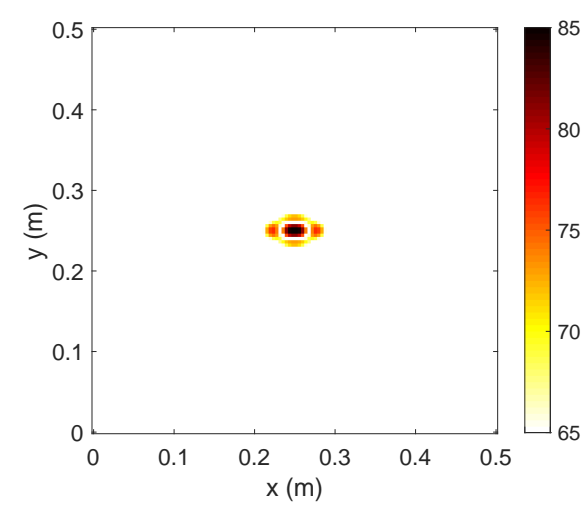

Fig. 6. Estimated pressure fields $\left(\mathrm{dB}\right.$ ref $1 \mathrm{~N} / \mathrm{m}^{2}$ ) of the orthotropic composite plate integrated from 1 to $10 \mathrm{kHz}$. FAT (a). CFAT (b).

(a) FAT

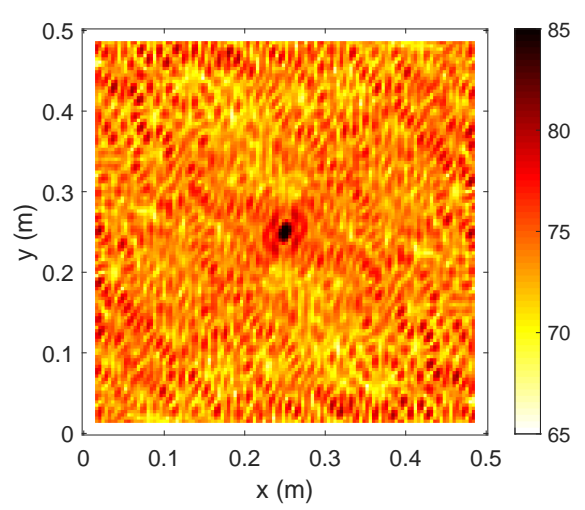

(b) CFAT

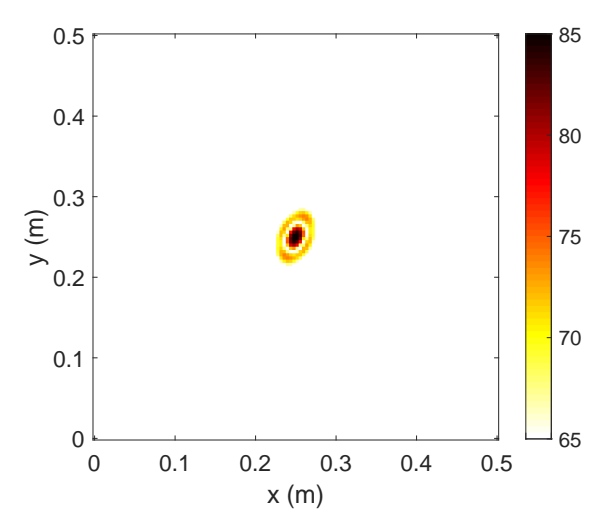

Fig. 7. Estimated pressure fields $\left(\mathrm{dB}\right.$ ref $\left.1 \mathrm{~N} / \mathrm{m}^{2}\right)$ of the laminated composite plate integrated from 1 to $10 \mathrm{kHz}$. FAT (a). CFAT (b).

\section{Application of CFAT in the presence of noise}

As stated by Ablitzer et al. [30], in the case of noisy measurements, the finite difference scheme can strongly increase the amplitude of noise. This phenomenon can be observed at low frequency when the size of the scheme is small as compared to the wavelength. In practice, this problem appears when the parameter $n$ becomes greater than 4 approximately [3123]. However, as presented in section 3.1, this value of 4 points per wavelength corresponds also to the high frequency limit of the FAT method beyond which one the bias error of the scheme becomes significant. Then, the amplification of noise is a problem for the FAT method in the frequency band defined by $n>4$. To tackle this issue, FAT uses a wavenumber low-pass filtering (not developed for orthotropic or laminated structures) that will be not presented in this paper. 


\subsection{Regularisation technique of CFAT: adjustment of the scheme as function of frequency}

The CFAT method can handle, with the correction factors, values of $n$ greatly lower than 4 up to the spatial limit of Shannon defined by 2 points per wavelength. Then, the bias error of the scheme as well as the amplification of noise can be controlled in a frequency band defined by $4>n>2$, the response of CFAT behaving as a low pass filter in wavenumber. Leclère et al. [23] proposed to adapt the size of the scheme as function of frequency to keep values of $n$ in this optimal interval. This approach, developed for isotropic structures, is extended here for the general anisotropic case. The measured mesh being Cartesian, the condition is applied in the directions $x$ and $y$ individually:

$$
4>n_{x}>2 \quad ; \quad 4>n_{y}>2,
$$

This condition is applied by adapting the size of the scheme as function of frequency:

$$
\Delta_{x}^{\prime}(f)=p_{x}(f) \Delta_{x} \quad ; \quad \Delta_{y}^{\prime}(f)=p_{y}(f) \Delta_{y}
$$

where $p_{x}$ and $p_{y}$ are natural integers depending on the frequency. Figure 8 presents an example of adapted scheme for $\left(p_{x}, p_{y}\right)=(2,1)$.

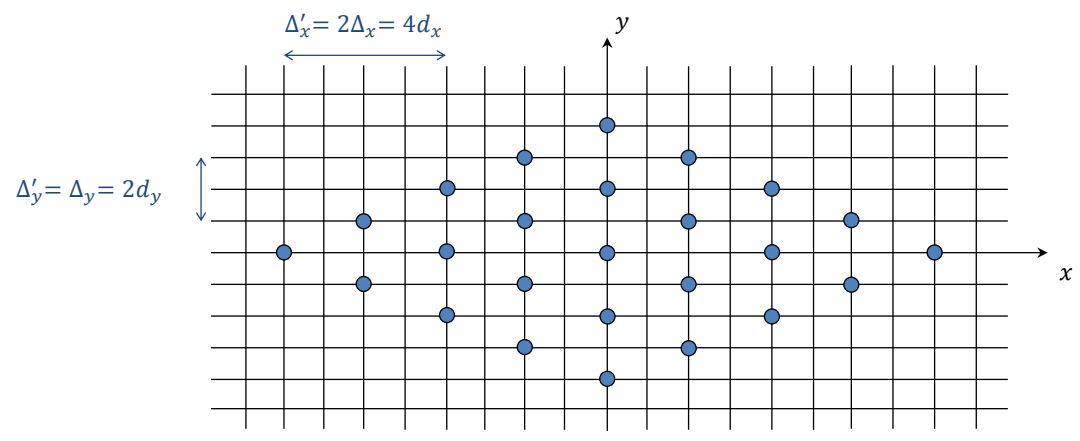

Fig. 8. Adapted finite difference scheme of CFAT for $\left(p_{x}, p_{y}\right)=(2,1)$.

\subsection{Noisy numerical data}

In order to illustrate the efficiency of this regularisation technique, a white noise has been added on the numerical displacement field numerically calculated by Nastran in section 3 and has been normalized to obtain a Signal to Noise Ratio of $20 \mathrm{~dB}$. The regularisation technique has been applied following the condition (24). The frequency dependency of $\left(n_{x}, n_{y}\right)$ and $\left(p_{x}, p_{y}\right)$ is drawn in Figure 9 for both plates. Figure 10 presents the identified load pressure field integrated in the whole frequency band. The pressure levels outside the loaded area are amplified by the effect of noise but are still negligible as compared to the pressure levels inside the loaded area thanks to the regularisation technique. 
(a) Orthotropic plate

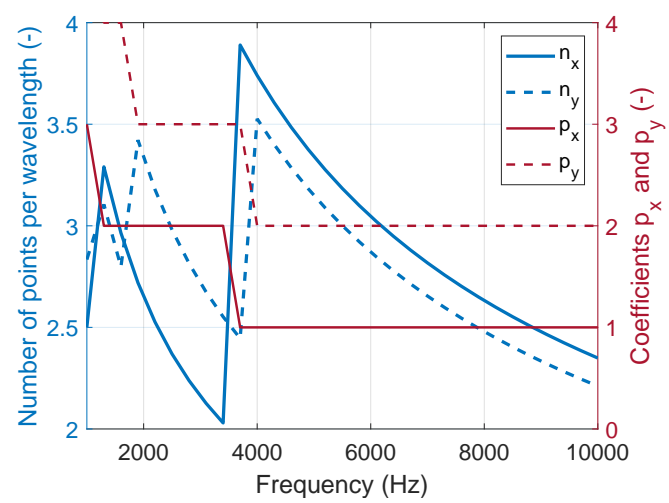

(b) Laminated plate

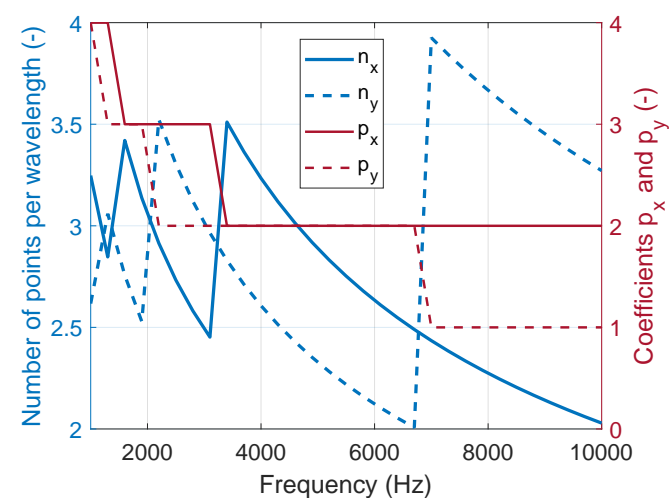

Fig. 9. Evolution of the number of points per wavelength $\left(n_{x}, n_{y}\right)$ and the coefficients $\left(p_{x}, p_{y}\right)$ as function of frequency by adapting the size of the scheme in CFAT. Orthotropic plate (a). Laminated plate (b).

(a) Orthotropic plate

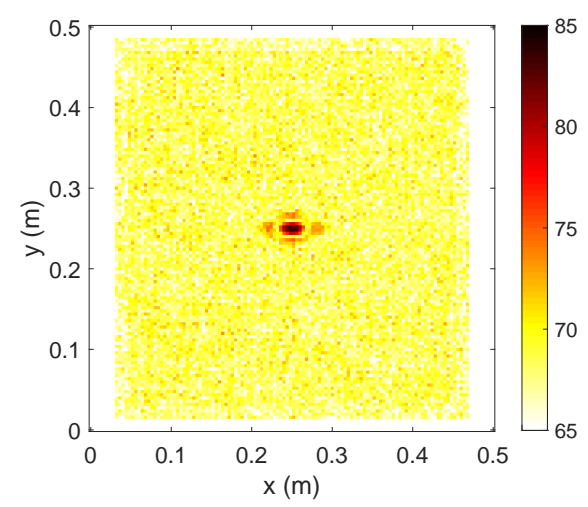

(b) Laminated plate

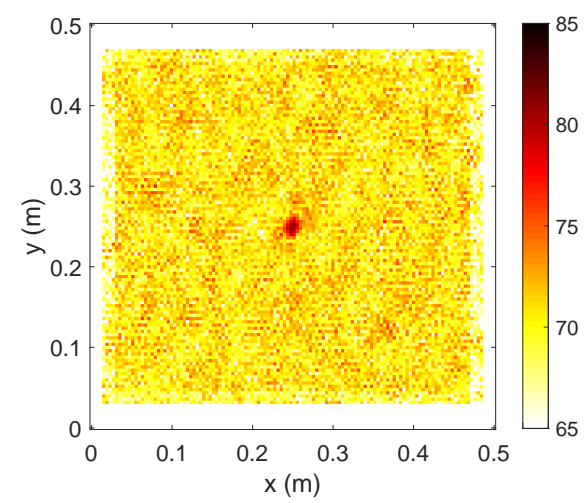

Fig. 10. Load distribution ( $\mathrm{dB}$, ref $1 \mathrm{~N} / \mathrm{m}^{2}$ ) estimated by CFAT applied on noisy numerical data (integrated from 1 to $10 \mathrm{kHz}$ ). Orthotropic plate (a). Laminated plate (b).

Figure 11 presents the force spectrum identified by CFAT inside and outside the load area for both plates. Forces are calculated by spatially integrating the pressure field in the studied area. The first spectrum (black dots) corresponds to the input force applied on the structure at the excitation point while the second (red dots) represents the residual forces. We can observed that the input force is close to the theoretical force of $1 \mathrm{~N}$ on the whole frequency range and the residual forces are approximately $10 \mathrm{~dB}$ lower.

\section{Experimental application}

This last section deals with the experimental validation of the CFAT method for laminated structures. Measurements have been conducted on carbon fibers composite plates defined by the same characteristics as the plates numerically studied 
(a) Orthotropic plate

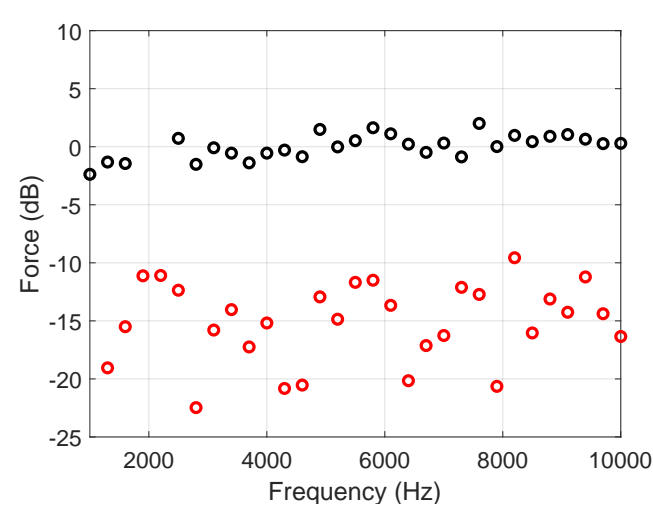

(b) Laminated plate

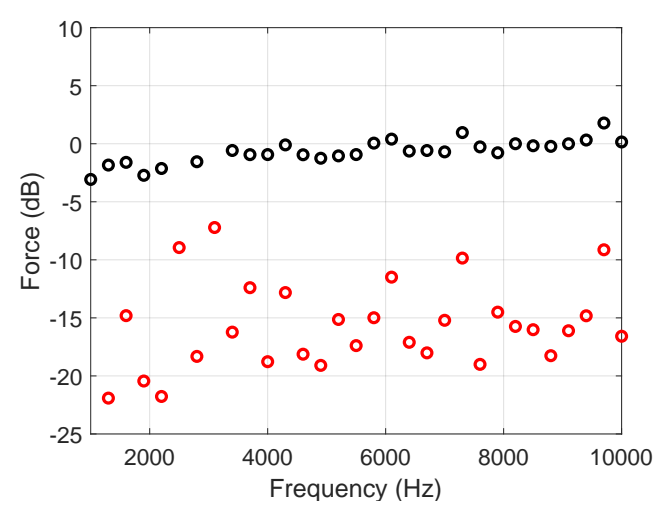

Fig. 11. Force spectrum (dB, ref $1 \mathrm{~N}$ ) estimated by CFAT inside (black dots) and outside (red dots) the loaded area. (a): Orthotropic plate, (b): Laminated plate.

in the previous sections. The structures, freely hung up by a rubber band to a frame, have been excited by a shaker, using white noise from 1 to $10 \mathrm{kHz}$. The vibration velocity fields of the plates were measured by means of a scanning Laser Doppler Vibrometer (Polytec, PSV-400) across the red area as indicated in Figure 12, A measurement mesh of $55 \times 57$ points has been used. The spacing between consecutive points of the mesh in the $x$ and $y$ directions is $d_{x}=d_{y}=5.3 \mathrm{~mm}$. With this mesh, the spatial limit of Shannon is verified up to $7 \mathrm{kHz}$ for the orthotropic plate and $10 \mathrm{kHz}$ for the anisotropic plate. Note that foam was added on the plate edges (see Figure 12), in order to attenuate the reverberation time of the structure. By doing so, the time window used for the FFT can be significantly shortened, as well as the full measurement time, that can be quite long for such a scan (several hours for more than 3000 measurement points).
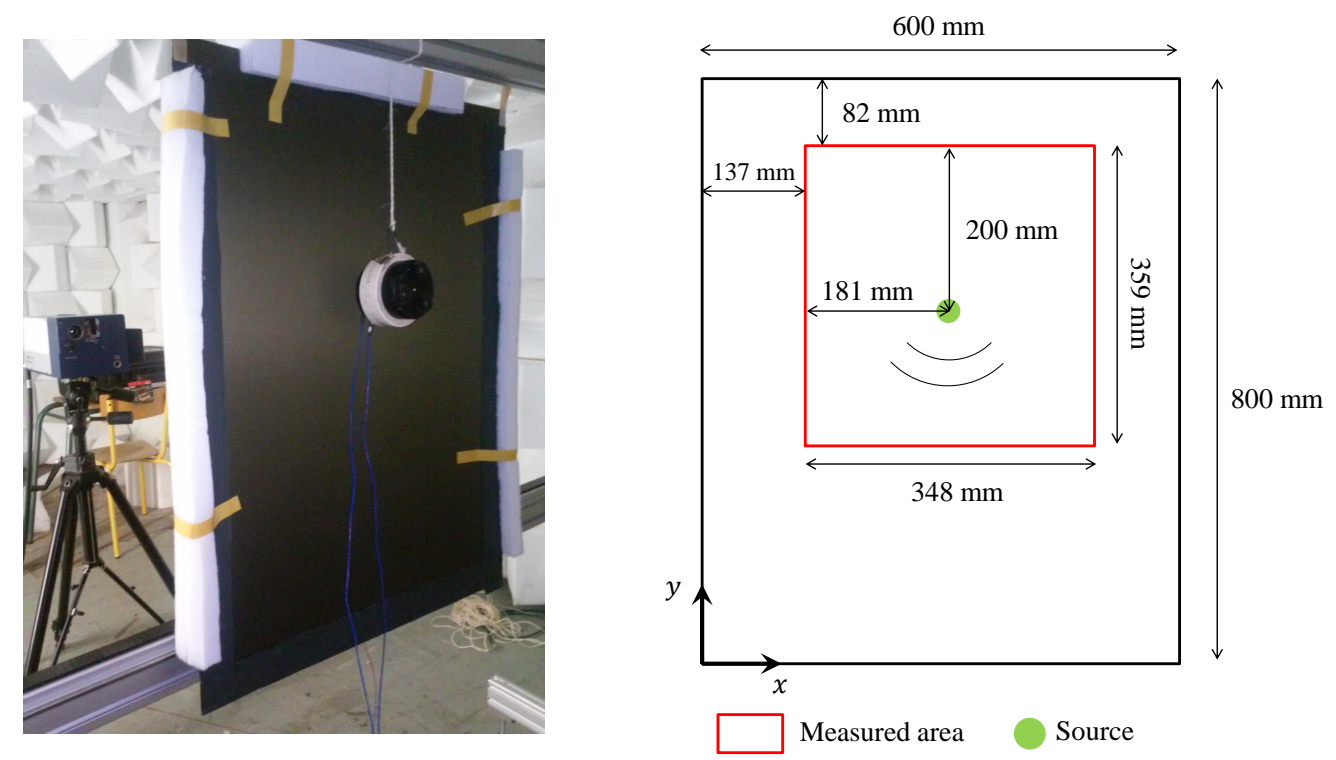

Fig. 12. Experimental setup (identical to [28])

Figure 13 presents the reconstructed pressure field for both plates. In the non-loaded 
area, the noise level is less important than in the results of the numerical simulations in the previous section.

(a) Orthotropic plate

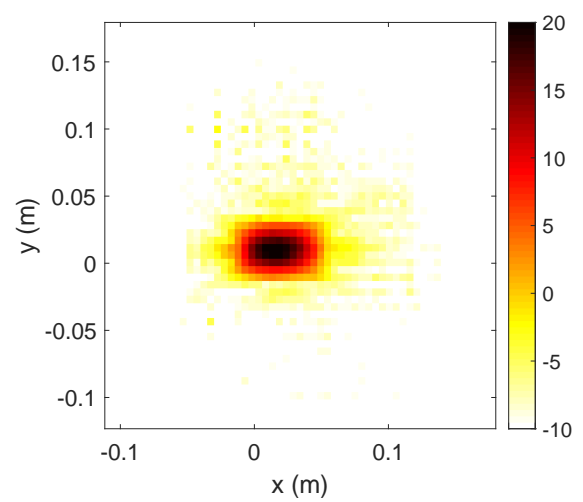

(b) Laminated plate

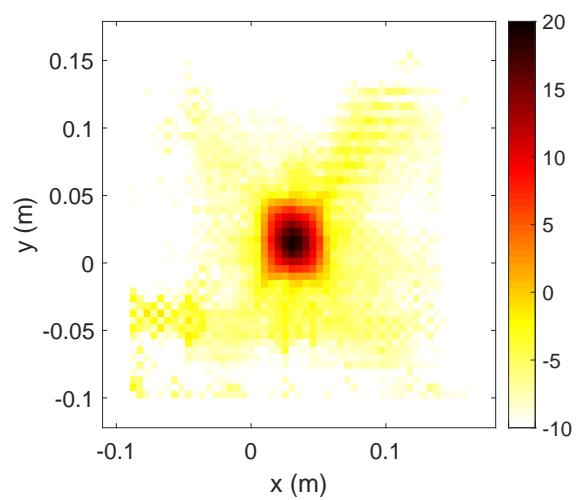

Fig. 13. Load distribution ( $\mathrm{dB}$, ref $\left.1 \mathrm{~N} / \mathrm{m}^{2}\right)$ estimated by CFAT applied on experimental data. (a): Orthotropic plate (integrated from 1 to $7 \mathrm{kHz}$ ), (b): Laminated plate (integrated from 1 to $10 \mathrm{kHz}$ ).

Figure 14 compares the spectra of the input and residual forces identified by CFAT with a recalculated force $F_{c}$ for both plates. This third force has been calculated using the theoretical admittance of an infinite plate $Y_{c}$ (following Skudrzyk's mean value theory [32] applied for orthotropic plates [33,34|35]) and the measured velocity at the excitation point $w_{c}$ :

$$
F_{c}=\frac{w_{c}}{Y_{c}}
$$

with:

$$
Y_{c}=\frac{\mathcal{F}}{4 \pi \sqrt{\rho h \sqrt{D_{11} D_{22}}}}
$$

where $\mathcal{F}$ represents the elliptic integral of first order:

$$
\mathcal{F}=\int_{0}^{\frac{\pi}{2}}\left(1-0.5\left(1-\frac{D_{12}}{2 \sqrt{D_{11} D_{22}}}\right) \sin ^{2}(\theta)\right)^{-0.5} d \theta
$$

Note that the input admittance of the plate has not been measured since it has been observed that the structure was so light that the measured force was mainly generated by inertial effects of the force sensor itself (almost the same signal was obtained with or without connection to the structure).

The injected force follows particularly well the recalculated force for both plates. As expected, the residual forces are insignificant compared to the injected forces. For the laminated plate, the abrupt increasing of the residual force level around 5.5 $\mathrm{kHz}$ is due to the condition (24) which is not necessarily verified in all directions for this type of plate. 
(a) Orthotropic plate

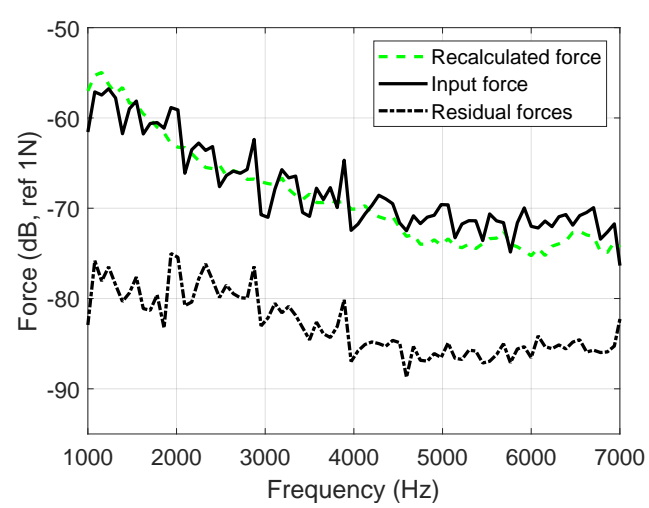

(b) Laminated plate

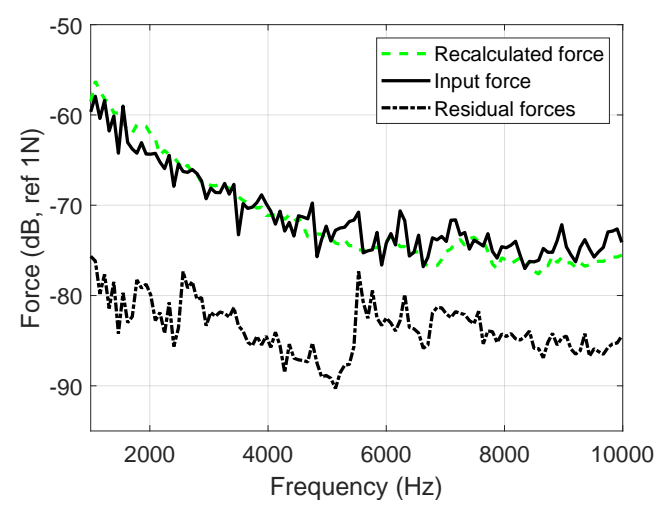

Fig. 14. Force spectrum (dB, ref $1 \mathrm{~N})$ estimated by CFAT inside (black solid line) and outside (black dot-dashed line) the loaded area and compared to a recalculated force (green dashed line). (a): Orthotropic plate, (b): Laminated plate.

\section{Conclusion}

This work details the extension of the CFAT [22] (Corrected Force Analysis Technique) method for source identification on laminated composite plates. The equation of motion of such structures is defined by 5 spatial derivatives under the thin plate theory and is estimated by FAT methodologies using a finite difference scheme [25]. The main novelty of this paper consists in using 5 correction factors to attenuate the bias error of this scheme. These coefficients are calculated using a least square method which gives a regular distribution of the attenuation in the wavenumber domain. With this correction, the amplification of the pressure level due to the bias error of the scheme is attenuated outside the loaded area of the reconstructed field.

In the case of noisy measurements, CFAT can control the amplification of noise in a given frequency band defined by the size of the scheme. Then, a regularisation technique [23] based on the adaptation of the size of the scheme as function of frequency has been developed to handle measurement uncertainties in the whole frequency band. Since the studied structures are anisotropic, we propose to apply this technique in $x$ and $y$ directions separately. The extension of the CFAT methodology as well as the regularisation technique have been applied on measured displacement fields of carbon fiber composite plates. The experimental results show a good agreement between the estimated force spectrum and a recalculated force spectrum based on the theoretical admittance of an infinite plate.

It can be noticed that the regular measured mesh constitutes a limitation for the method since the ideal number of points per wavelength needed in the regularisation process is not respected in all directions. Futhermore, the size and the spacing between the points of the measurement mesh define the lower and upper limits of the frequency band of interest, respectively. 
In a further step, this method could be used for material characterization of composite structures assuming that the reconstructed pressure field is null in an area far from the source [30[24]. Another perspective could also be to adapt CFAT for thick structures whose flexural behaviour is dominated by the shearing effect in high frequencies (as already introduced by Wassereau et al. [26] on sandwich beams).

\section{Acknowledgments}

This work has been initiated during the PhDs of the first author at the LVA, for which he was sponsored by the French Ministry of Research. This work was performed within the framework of the Labex CeLyA of Université de Lyon, operated by the French National Research Agency (ANR-10-LABX- 0060/ ANR-11-IDEX0007).

\section{References}

[1] K.S. Stevens. Force identification problems: an overview. Proceedings of SEM Spring Conference on Experimental Mechanics, pages 838-844, 1987.

[2] J. Sanchez and H. Benaroya. Review of force reconstruction techniques. Journal of Sound and Vibration, 333(14):2999 - 3018, 2014.

[3] J.A. Fabunmi. Effects of structural modes on vibratory force determination by the pseudoinverse technique. AIAA Journal, 24:504-509, 1986.

[4] W. Hendricx and D. Vandenbroeck. Suspension analysis in view of road noise optimization. In SAE Technical Paper. SAE International, 1993.

[5] P. Mas, P. Sas, and K. Wyckaert. Indirect force identification based upon impedance matrix inversion : a study on statistical and deterministical accuracy. Proceedings of ISMA 19, 1994.

[6] D. D. Jackson. Interpretation of inaccurate, insufficient and inconsistent data. Geophysical Journal of the Royal Astronomical Society, 28(2):97-109, 1972.

[7] W. Menke. Geophysical Data Analysis: Discrete Inverse Theory. Academic Press, Orlando, 1984.

[8] P. Guillaume, E. Parloo, and G. De Sitter. Source identification from noisy response measurements using an iterative weighted pseudo-inverse approach. In proceedings of ISMA, volume 4, 2002.

[9] W.M. To and D.J. Ewins. The role of the generalized inverse in structural dynamics. Journal of Sound and Vibration, 186(2):185 - 195, 1995. 
[10] E. Jacquelin, A. Bennani, and P. Hamelin. Force reconstruction: analysis and regularization of a deconvolution problem. Journal of Sound and Vibration, 265(1):81 $-107,2003$.

[11] M. Aucejo. Structural source identification using a generalized tikhonov regularization. Journal of Sound and Vibration, 333(22):5693 - 5707, 2014.

[12] D.U. Noiseux. Measurement of power flow in uniform beams and plates. The Journal of the Acoustical Society of America, 47(1B):238-247, 1970.

[13] G. Pavic. Measurement of structure borne wave intensity, part i: Formulation of the methods. Journal of Sound and Vibration, 49(2):221 - 230, 1976.

[14] Y. Zhang and J.A. Mann. Measuring the structural intensity and force distribution in plates. The Journal of the Acoustical Society of America, 99(1):345-353, 1996.

[15] F. Pierron and M. Grédiac. The virtual fields method. Extracting constitutive mechanical parameters from full-field deformation measurements. Springer-Verlag, 2012.

[16] A. Berry, O. Robin, and F. Pierron. Identification of dynamic loading on a bending plate using the virtual fields method. Journal of Sound and Vibration, 333(26):7151 7164, 2014.

[17] A. Berry and O. Robin. Identification of spatially correlated excitations on a bending plate using the virtual fields method. Journal of Sound and Vibration, 375:76 - 91, 2016.

[18] P. O'Donoughue, O. Robin, and A. Berry. Time-resolved identification of mechanical loadings on plates using the virtual fields method and deflectometry measurements. Strain, 54, 122017.

[19] C. Pézerat and J.-L. Guyader. Two inverse methods for localization of external sources exciting a beam. Acta Acustica, 3:1-10, 1995.

[20] C. Pézerat and J.-L. Guyader. Force analysis technique : reconstruction of force distribution on plates. Acustica United with Acta Acustica, 86:322-332, 2000.

[21] M.S. Djamaa, N. Ouella, C. Pézerat, and J.-L. Guyader. Reconstruction of a distributed force applied on a thin cylindrical shell by an inverse method and spatial filtering. Journal of Sound and Vibration, 301:560-575, 2007.

[22] Q. Leclère and C. Pézerat. Vibration source identification using corrected finite difference schemes. Journal of Sound and Vibration, 331(6):1366-1377, 2012.

[23] Q. Leclère, F. Ablitzer, and C. Pézerat. Identification of loads of thin structures with the corrected force analysis technique: An alternative to spatial filtering regularization. Proceedings of ISMA 2014, 2014.

[24] Q. Leclère, F. Ablitzer, and C. Pézerat. Practical implementation of the Corrected Force Analysis Technique to identify the structural parameter and load distributions. Journal of Sound and Vibration, 351:106-118, 2015. 
[25] F. Ablitzer, C. Pézerat, B. Lascoup, and J. Brocail. Identification of the flexural stiffness parameters of an orthotropic plate from the local dynamic equilibrium without a priori knowledge of the principal directions. Journal of Sound and Vibration, 404:31-46, 2017.

[26] T. Wassereau, F. Ablitzer, C. Pézerat, and J.L. Guyader. Experimental identification of flexural and shear complex moduli by inverting the timoshenko beam problem. Journal of Sound and Vibration, 399:86 - 103, 2017.

[27] J.M. Berthelot and J.M. Cole. Composite Materials: Mechanical Behavior and Structural Analysis. Mechanical Engineering Series. Springer New York, 1998.

[28] F. Marchetti, K. Ege, Q. Leclère, and N.B. Roozen. On the structural dynamics of laminated composite plates and sandwich structures; modelling and experiment. Submitted to Journal of Sound and Vibration, 2019.

[29] F. Marchetti, K. Ege, and Q. Leclère. Localisation and identification of sources on laminated structures : Extension of cfat method. In NOVEM, 2018.

[30] F. Ablitzer, C. Pézerat, J.-M. Génevaux, and J. Bégué. Identification of stiffness and damping properties of plates by using the local equation of motion. Journal of Sound and Vibration, 333(9):2454 - 2468, 2014.

[31] Q. Leclère and C. Pézerat. Time domain identification of loads on plate-like structures using an array of acoustic velocity sensors. In proceedings of Acoustics'08, 2008.

[32] E.J. Skudrzyk. The mean-value method of predicting the dynamic-response of complex vibrators. Journal of the Acoustical Society of America, 67(4):1105-1135, 1980 .

[33] J. P. D. Wilkinson. Modal densities of certain shallow structural elements. Journal of the Acoustical Society of America, 43(2):245-251, 1968.

[34] K. Ege. The piano soundboard - Modal studies in the low-and the mid-frequency range. Phd thesis in french, École Polytechnique, December 2009.

[35] X. Boutillon and K. Ege. Vibroacoustics of the piano soundboard: Reduced models, mobility synthesis, and acoustical radiation regime. Journal of Sound and Vibration, 332(18):4261-4279, 2013. 


\section{List of Figures}

$1 \quad$ Finite difference scheme of FAT and CFAT for the general anisotropic case. 5

2 Wavenumber responses (dB) of FAT (a) and CFAT (b) with

\begin{tabular}{|ll|}
\hline numerical correction factors (100 equations) for orthotropic \\
\hline \hline flexural rigidities with $n_{x}=4.42$ and $n_{y}=2.29$. \\
\hline
\end{tabular}

3 Wavenumber responses (dB) of FAT (a) and CFAT (b) with \begin{tabular}{|ll|}
\hline numerical correction factors (100 equations) for laminated flexural \\
\hline \hline rigidities with $n_{x}=2.61$ and $n_{y}=3.87$. \\
\hline
\end{tabular}

4 Relative values of the correction factor at $10 \mathrm{kHz}$ as function of the number of equations. (a): orthotropic plate, (b): laminated plate. $\quad 11$

$5 \quad$ Number of points per wavelength (a) and correction factors (b) calculated for the laminated plate with $\Delta_{x}=\Delta_{y}=4 \mathrm{~mm}$.

6 Estimated pressure fields $\left(\mathrm{dB}\right.$ ref $\left.1 \mathrm{~N} / \mathrm{m}^{2}\right)$ of the orthotropic composite plate integrated from 1 to $10 \mathrm{kHz}$. FAT (a). CFAT (b). 12

$7 \quad$ Estimated pressure fields $\left(\mathrm{dB}\right.$ ref $\left.1 \mathrm{~N} / \mathrm{m}^{2}\right)$ of the laminated composite plate integrated from 1 to $10 \mathrm{kHz}$. FAT (a). CFAT (b). 12

$8 \quad$ Adapted finite difference scheme of CFAT for $\left(p_{x}, p_{y}\right)=(2,1)$. $\quad 13$

9 Evolution of the number of points per wavelength $\left(n_{x}, n_{y}\right)$ and the coefficients $\left(p_{x}, p_{y}\right)$ as function of frequency by adapting the size $\begin{array}{ll}\text { of the scheme in CFAT. Orthotropic plate (a). Laminated plate (b). } & 14\end{array}$

10 Load distribution $\left(\mathrm{dB}\right.$, ref $\left.1 \mathrm{~N} / \mathrm{m}^{2}\right)$ estimated by CFAT applied on noisy numerical data (integrated from 1 to $10 \mathrm{kHz}$ ). Orthotropic plate (a). Laminated plate (b).

11 Force spectrum $(\mathrm{dB}$, ref $1 \mathrm{~N})$ estimated by CFAT inside (black dots) and outside (red dots) the loaded area. (a): Orthotropic plate, (b): Laminated plate.

12 Experimental setup (identical to [28])

13 Load distribution $\left(\mathrm{dB}\right.$, ref $\left.1 \mathrm{~N} / \mathrm{m}^{2}\right)$ estimated by CFAT applied on experimental data. (a): Orthotropic plate (integrated from 1 to 7 $\mathrm{kHz}$ ), (b): Laminated plate (integrated from 1 to $10 \mathrm{kHz}$ ). 
14 Force spectrum $(\mathrm{dB}$, ref $1 \mathrm{~N})$ estimated by CFAT inside (black solid line) and outside (black dot-dashed line) the loaded area and compared to a recalculated force (green dashed line). (a): Orthotropic plate, (b): Laminated plate. 


\section{List of Tables}

1 Characteristics of one layer of the simulated multilayer laminated plates.

2 Equivalent flexural rigidities of the $0^{\circ} / 0^{\circ} / 0^{\circ} / 0^{\circ}$ composite plate at $10 \mathrm{kHz}$, according to [28].

3 Equivalent flexural rigidities of the $60^{\circ} /-60^{\circ} /-60^{\circ} / 60^{\circ}$ composite plate at $10 \mathrm{kHz}$, according to [28]. 